Itinéraires Itinéraires

Littérature, textes, cultures

2012-3 | 2013

Lire les villes marocaines

\title{
L'hétérotopie tangéroise de Tahar Ben Jelloun
}

\section{Christiane Albert et Marc Kober}

\section{OpenEdition}

\section{Journals}

Édition électronique

URL : http://journals.openedition.org/itineraires/941

DOI : 10.4000/itineraires.941

ISSN : 2427-920X

Éditeur

Pléiade

Édition imprimée

Date de publication : 1 juillet 2013

Pagination : 47-56

ISBN : 978-2-343-01183-7

ISSN : $2100-1340$

Référence électronique

Christiane Albert et Marc Kober, «L'hétérotopie tangéroise de Tahar Ben Jelloun », Itinéraires [En ligne], 2012-3 | 2013, mis en ligne le 01 décembre 2012, consulté le 19 avril 2019. URL : http://

journals.openedition.org/itineraires/941 ; DOI : 10.4000/itineraires.941

\section{(c) ())}

Itinéraires est mis à disposition selon les termes de la licence Creative Commons Attribution - Pas d'Utilisation Commerciale - Pas de Modification 4.0 International. 


\title{
L’hétérotopie tangéroise de Tahar Ben Jelloun
}

\begin{abstract}
The representation of Tangier as Tahar Ben Jelloun draws up in "Tanger-la-trahison," "Tanger porte de l'Afrique" and Partir has nothing subjective, but rather contributes to the Tangierian heterotopia made up by all the literature written on the city. It can be read as a response to Western literary representations of Tangier, drawn up since the $19^{\text {th }}$ century, which reduce the description of the city to some of its elements: gambling dens, traffics, corruption and prostitution. However, although the intention of Tahar Ben Jelloun is to redeem the city from Western disparaging views, Tangier is still depicted as the city of all betrayals, even in Partir, written twenty-five years after "Tanger-la-trahison." It does not operate any break with the pre-existing representations, even if the phenomenon of immigration has been taken into account. Tangier remains a space heavily vested in imagination and legend.
\end{abstract}

Keywords : Tangier, heterotopia, myth, immigration, space

Mots clés : Tanger, hétérotopie, mythe, immigration, espace

Dès ses premiers textes, la ville de Tanger est représentée dans l'œuvre de Tahar Ben Jelloun. Elle est au centre de « Tanger-la-trahison », qui est une sorte de poème/récit publié en 1973, inclus dans Harrouda ${ }^{1}$, et de « Tanger porte de l'Afrique » qui fait partie du recueil : Les amandiers sont morts de leurs blessures ${ }^{2}$ publié en 1976. On la trouve aussi représentée dans Partir $^{3}$ publié en 2006, dont l'essentiel se passe à Tanger. Cependant, malgré les liens affectifs qui unissent l'écrivain à cette ville où il a vécu, la représentation littéraire qu'il en donne n'est pas entièrement subjective. Elle se réfère davantage à d'autres représentations littéraires préexistantes de la ville. Au point que l'ensemble des textes publiés sur Tanger finit par

1. Paris, Denoël, 1973.

2. Tahar Ben Jelloun, Les amandiers sont morts de leurs blessures [1976], Paris, Maspero 1999.

3. Tahar Ben Jelloun, Partir, Paris, Gallimard, 2006. 
constituer un seul et même corpus qu'on peut lire comme une hétérotopie, dans le sens où Foucault la définit:

Il y a également, et ceci probablement dans toute culture, dans toute civilisation, des lieux réels, des lieux effectifs, des lieux qui sont dessinés dans l'institution même de la société, et qui sont des sortes de contre-emplacements, sortes d'utopies effectivement réalisées dans lesquelles les emplacements réels, tous les autres emplacements réels que l'on peut trouver à l'intérieur de la culture sont à la fois représentés, contestés et inversés, des sortes de lieux qui sont hors de tous les lieux, bien que pourtant ils soient effectivement localisables. Ces lieux, parce qu'ils sont absolument autres que tous les emplacements qu'ils reflètent et dont ils parlent, je les appellerai, par opposition aux utopies, les hétérotopies; $[\ldots]^{4}$.

L'hétérotopie constitue ainsi un espace du dehors où peuvent se structurer des discours et des regards distanciés sur le monde. Dans cette perspective, l'espace littéraire tangérois pourrait se définir comme un espace hétérotopique, un espace « autre » mettant cependant en jeu des questions bien réelles d'identité, d'altérité et d'exotisme.

Aussi, et cela sera notre hypothèse de départ, les trois textes de Tahar Ben Jelloun qui concernent explicitement Tanger peuvent se lire comme une réponse aux représentations littéraires occidentales de Tanger, élaborées depuis le $\mathrm{XIX}^{\mathrm{e}}$ siècle, qui réduisent la ville à certains de ses éléments : les tripots, les trafics, la corruption, la prostitution. L'intention de Tahar Ben Jelloun est de répondre à ce regard réducteur que les Occidentaux portent sur la ville en éclairant les raisons historiques et sociales qui expliquent ces pratiques. Il n'en demeure pas moins que, ce faisant, la représentation qu'il élabore de Tanger ne marque aucune rupture avec celles de ses prédécesseurs : la ville qu'il dépeint demeure toujours un repaire de traîtres, de voleurs et de prostitués des deux sexes et contribue de ce fait à nourrir le mythe littéraire de Tanger. Un élément nouveau intervient cependant dans Partir qui prend en compte le phénomène de l'immigration, absent des représentations élaborées dans les années 1980. Il se produit alors une redisposition des topoï constitutifs du mythe qui l'oriente différemment. L'immigration introduit en effet dans la représentation des enchevêtrements d'espaces entre lesquels les personnages circulent. Ces parcours cheminatoires s'inscrivent dans une autre spatialité que celle de la ville des années 1980, celle de « Tanger-la-trahison » ou de « Tanger porte de l'Afrique », qui était encore une ville cartographiable, limitée géographiquement à une portion de l'espace. Dans Partir, sous l'effet des phénomènes liés à la migration, l'espace délocalisé de Tanger construit une ville

4. Michel Foucault, «Des espaces autres», Architecture, Mouvement, Continuité, $\mathrm{n}^{\circ}$ 5, 1984, p. 46-49, p. 47. Il s'agit à l'origine d'une conférence prononcée au Cercle d'études architecturales, le 14 mars 1967. Elle a été reprise dans le volume Dits et Écrits 1, 1954-1975, Paris, Gallimard, 2001. 
« transhumante ${ }^{5}$ » qui se prolonge jusqu'à certains quartiers de Malaga ou de Madrid que plus rien ne distingue de Tanger. La prise en compte par le roman de ce phénomène nouveau de l'immigration rajoute ainsi une nouvelle strate dans le continuum spatio-temporel de la représentation. Mais paradoxalement, cette nouvelle strate ne remet pas en question l'aspect mythique de la ville qui constitue toujours un espace hétérotopique, un espace autre.

\section{La déréalisation de Tanger}

Il faut cependant préciser que parler de déréalisation ne signifie pas que la ville dont il est question dans les textes de notre corpus soit une ville de fiction qui ne renvoie à aucun référent réel. Le Tanger de Tahar Ben Jelloun s'inscrit géographiquement dans le continent africain comme l'atteste le titre du poème "Tanger porte de l'Afrique ». La ville est en effet située à la confluence de la Méditerranée et de l'Atlantique, dans sa proximité avec l'Espagne, « quatorze kilomètres », fréquemment évoqués dans le texte. Mais la ville est aussi décrite, avec ses différents espaces nommément cités : le Socco, le port, les plages, la Montagne qui permettent de suivre les déambulations urbaines des personnages à partir de références précises, vérifiables sur n'importe quel plan de la ville. De plus, cette volonté de représenter la ville de façon réaliste prend en compte sa réalité sociale et économique en décrivant les quartiers riches, la misère mais aussi le chômage, les abus de pouvoir et les injustices que dénonce Tahar Ben Jelloun. Cette dénonciation, souvent proche du réquisitoire, correspond à l'engagement politique de l'écrivain, explicite dans tous ses textes, et notamment dans Partir qui évoque l'exploitation des enfants travaillant pour des salaires de misère dans des usines de crevettes où ils finissent par mourir à cause du froid qui y règne ou encore le chômage des jeunes diplômés pour lesquels la prostitution et l'exil sont les seules alternatives.

Cependant malgré son aspect réaliste, on peut considérer que la représentation qu'élabore Tahar Ben Jelloun de Tanger renvoie autant à la fiction qu'au réel car le texte est aussi - et sans ambiguïté - une réponse à « tous les porteurs de signes étrangers » que le récitant interpelle dans « Tanger-latrahison » en se référant explicitement à Delacroix, Burroughs ou Kerouac : « si vous désirez trahir dans ma ville déchiffrez d'abord l'écriture des migrations premières ${ }^{6} \gg$. Pour permettre ce déchiffrement, le texte commence par évoquer le passé glorieux et légendaire de la ville, occulté par la vision orientaliste et occidentale. Il se réfère d'abord à Tarik bnû Zaïyad ${ }^{7}$, conquérant de l'Espagne. Il rappelle ensuite la défaite de Ferdinand Ir du Portugal qui

5. Michel de Certeau, L'Invention du quotidien, t. I, Arts de faire, Paris, Gallimard, 1990.

6. Tahar Ben Jelloun, Partir, op. cit., p. 115.

7. «Tarik bnû Zaïyad - un guerrier plus doué qu'Hercule - a fait de la mer notre écart. » (Harrouda, op. cit., p. 120.) 
échoua à conquérir la ville et fut réduit en esclavage, puis celle de Charles II et il s'achève par l'évocation d'Abd el Krim al Khattabi, grande figure de la guerre anticolonialiste du Rif, qui battit les Espagnols avant d'être vaincu par les Français à la suite de trahisons qui eurent pour cadre Tanger, « lieu - dit le récitant - de la trahison - trahison et corruption des hommes - s'y retrouvaient tous ceux qui élaboraient tranquillement dans des chambres d'hôtel des plans pour piller les mines du Rif et asservir le peuple ${ }^{8} \gg$. Il s'agit là d'événements historiques vérifiables qui permettent de réhabiliter la ville face au regard dépréciateur et orientaliste des Occidentaux (la référence à certains passages du journal de Delacroix est bien sûr très significative). Cette évocation du passé glorieux de la ville et de son histoire peut s'interpréter dans une perspective postcoloniale comme une forme de résistance à l'impérialisme culturel de l'Occident. Elle permet aussi l'adjonction de nouvelles strates temporelles qui donnent de la profondeur à la représentation de Tanger puisque l'évocation commence au temps mythique de la légende d'Hercule et s'achève à l'époque contemporaine de l'écriture.

Mais la réponse de Tahar Ben Jelloun aux Occidentaux concerne aussi l'autre versant de la trahison qui fascinait tant Genet, et qui n'avait rien de politique : la prostitution. Elle était inséparable pour lui de l'érotisme qui prit à Tanger la forme d'un tourisme sexuel pratiqué par « les homosexuels voyageurs souvent riches » auxquels Tahar Ben Jelloun rappelle l'inégalité de l'échange : «l'un produit le plaisir l'autre donne de l'argent» et « la honte est à la charge de l'autre », l'étranger, qui ignore que la sodomie est considérée par les Marocains comme le comble de «l'humiliation». De ce fait, continue le récitant, il n'y a qu'une «illusion d'échange»: « le garçon pense faire honte à l'Occidental : il en est fier et il rit» ce qui constitue sa revanche. Quant à l'étranger, « il vit une certaine forme de culpabilité qu'il tend à masquer et à oublier en venant dans ces pays où la pratique est facile ${ }^{9} \gg$. On retrouvera dans Partir la même dénonciation du tourisme sexuel, avec les mêmes arguments puisque le héros du roman, Azel, se prostitue avec un riche Espagnol pour les mêmes raisons et avec les mêmes sentiments.

Mais il existe encore une autre forme de trahison dans «Tanger-latrahison » « la plus médiocre ${ }^{10} »$, celle d'une ville «travestie, maquillée aux couleurs hollywoodienne, avec la Kasba en carton-pâte et la grande mosquée transparente. Tanger mal aimée, mal écrite. La nommer, la représenter, c'était la détruire ${ }^{11}$ ». Le poème s'achève par le constat que « la vie faite se défait à Tanger ${ }^{12}$ » et semble prendre acte de la fin de la légende tangéroise :

8. Tahar Ben Jelloun, Harrouda, op. cit., p. 132.

9. Ibid., p. 139-140.

10. Ibid., p. 142.

11. Ibid.

12. Ibid., p. 135. 


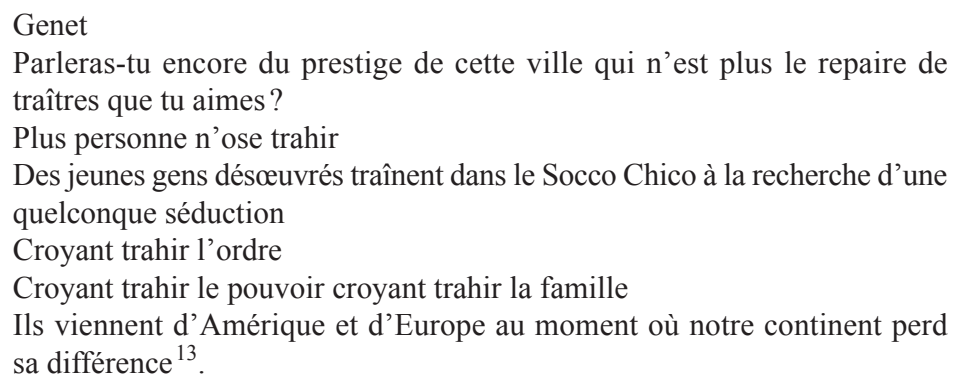

Mais Tahar Ben Jelloun n'a cependant pas fini de régler ses comptes avec les « porteurs de signes étrangers » puisque le réquisitoire se poursuit dans Partir écrit vingt-cinq ans après « Tanger-la-Trahison » :

Ces gens-là, ils veulent tout des hommes et des femmes du peuple, les servant le jour, les niquant la nuit. Service complet et entre deux petits coups, une pipe de kif bien bourrée pour que l'Américain écrive! Il leur dit racontemoi ta vie, j'en ferai un roman; tu auras ton nom sur la couverture, tu ne pourras pas le lire mais ça ne fait rien, tu es écrivain comme moi, sauf que toi on dira : c'est un écrivain analphabète, c'est exotique, je veux dire étrange, mon $\mathrm{ami}^{14}$.

On reconnaît bien sûr sans peine Paul Bowles et sa femme ainsi que les écrivains marocains qui gravitaient autour d'eux, Mohamed Mrabet et Larbi Layachi. Mais au-delà de ces références précises, réel et fiction continuent de se confondre dans le roman où le mythe est à nouveau réactivé : Tanger reste toujours « la ville de tous les bandits et de tous les traîtres ${ }^{15}$ ». On continue à y trafiquer, à y trahir. Les passeurs, les dealers et les proxénètes ont remplacé la faune internationale du Tanger des années 1950, mais les jeunes gens pauvres, serveurs le jour et amants la nuit, continuent de vendre leur corps à de riches étrangers qui persistent à organiser dans la Montagne des fêtes somptueuses et extravagantes où les invités « ont l'air de s'être trompé d'époque » et où l'on croise toujours « une vieille princesse d'un pays lointain, un vieux ministre ou encore quelques stars sur le retour ${ }^{16} »$. Tout au plus peut-on observer un certain vieillissement des invités, mais le temps semble s'être arrêté dans ce Tanger du début $\mathrm{du} \mathrm{XXI}{ }^{\mathrm{e}}$ siècle, comme l'atteste la scène d'ouverture du roman qui se situe dans un café où de jeunes Marocains continuent à rêver en fumant du kif et en fantasmant sur la créature légendaire de Toutia ${ }^{17}$. L'hétérotopie tangéroise de Tahar Ben Jelloun se double ainsi d'une hétérochronie, que Foucault qualifie de découpage du temps, principe selon lui constitutif de

13. Ibid., p. 147.

14. Tahar Ben Jelloun, Partir, op. cit., p. 47.

15. Ibid., p. 112.

16. Ibid., p. 67.

17. « Toutia » qui n'est pas sans rappeler l'« Harrouda » du texte éponyme écrit en 1971. 
l'hétérotopie « [qui] se met à fonctionner à plein lorsque les hommes se trouvent dans une sorte de rupture absolue avec leur temps traditionnel ${ }^{18} »$.

Les paragraphes qui ouvrent le deuxième chapitre de Partir illustrent bien la rupture chronologique à l'œuvre dans l'hétérotopie tangéroise. En effet, le personnage central, Azel, tente de suturer les pans textiles et textuels de la ville par l'activité symbolique de la marche, comme s'il essayait de restaurer un ordre présent avec ses pieds.

Chaque fois qu'Azel quitte ce silence où aucune présence ne s'impose, il a froid. Quelle que soit la saison, son corps est secoué par un léger tremblement. Il sent le besoin de s'éloigner de la nuit, il refuse d'y entrer. Il marche dans la ville, ne parle à personne, s'imagine tailleur, couturier d'un genre à part, reliant les ruelles étroites aux larges avenues avec un fil blanc comme dans cette histoire que lui racontait sa mère quand il avait du mal à s'endormir. Il voulait savoir si Tanger était une djellaba d'homme ou un caftan de mariée, mais la ville avait tellement grossi qu'il avait renoncé à son idée ${ }^{19}$.

À partir de là, la métaphore vestimentaire de Tanger, tantôt djellaba, tantôt caftan, se termine de façon dysphorique par une absence de vêtement traditionnel. Si la ville est trop grosse, c'est sous l'effet d'un gonflement démographique et d'une urbanisation croissante qui ont fait éclater les limites de la ville traditionnelle. On peut penser aussi à la suralimentation, fléau des pays riches et industrialisés d'Europe et du Nouveau Monde, ou encore à l'enrichissement d'une fraction corrompue du pays, grâce à différents trafics, mais aussi par le détournement de la richesse produite par ceux qui sont exploités. La ville n'est plus qu' « [...] une de ces couvertures de laine synthétique que les émigrés rapportent de Belgique ${ }^{20} \gg$. Cette dernière image permet de revenir au thème de l'immigration vers l'Europe, tout en suggérant la perte des valeurs artisanales, du souci de l'authentique. La rupture avec le temps traditionnel est consommée, et le héros peut se mouvoir dans la dimension hétérotopique.

Car la couverture qui ne réchauffe pas, le trophée dérisoire de l'immigration, recouvre le corps devenu monstrueux de la ville moderne. Le narrateur note le décentrement de la ville en raison du changement de moyen de locomotion, avec pour conséquence la rupture entre la ville et son arrièrepays. Le terroir agricole qui entourait Tanger se reliait à cette ville par la marche des paysannes, par leurs itinéraires réguliers, et par leur simple présence au cœur de la cité. La ville est « décousue », " détissée » pour reprendre l'image de l'auteur. Azel, le tisseur au fil blanc, abandonne son travail de couture parce que sa ville est défaite, comme un vêtement est

18. Michel Foucault, «Des espaces autres », op. cit., p. 48. Il s'agit d'une remarque qui figure dans le quatrième principe de l'hétérotopie avancé par le philosophe.

19. Tahar Ben Jelloun, Partir, op. cit., p. 16.

20. Ibid. 
décousu, ou mal assemblé, à cause de la puissance des courants migratoires, dont Tanger serait la source et le passage obligé.

La ville était dissimulée sous ce tissu qui maintenait la chaleur sans pour autant chasser l'humidité. Elle n'avait plus de forme, plus de centre, mais des places, pas tout à fait rondes, d'où les voitures ont délogé les paysannes venues du Fahs vendre leurs fruits et légumes.

La ville changeait et les murs se fissuraient ${ }^{21}$.

Malgré ces transformations insidieuses de Tanger, le temps semble s'être arrêté et le mythe se nourrit de lui-même dans l'espace textuel, palimpseste du roman. Une rupture intervient cependant dans cette représentation mythique de Tanger qui déconstruit ce que le texte s'efforce de construire en faisant intervenir une nouvelle donnée, celle de l'immigration qui oriente différemment le mythe en opérant un désancrage spatial de la ville qui contribue à la déterritorialiser.

\section{La déterritorialisation de Tanger dans Partir}

L'immigration introduit dans Partir un processus, proche de la synecdoque, qui a pour effet d' « amplifier le détail et de miniaturiser 1'ensemble ${ }^{22} \gg$. On assiste en effet dans le roman à une sorte de contamination des caractéristiques propres de Tanger au reste du pays, les deux finissant par se confondre et former un seul et même continuum spatial où la corruption, les trafics, la prostitution et aussi la misère ne laissent pas d'autre issue aux habitants que l'exil.

Le Café Hafa, qui est décrit dans les premières pages est un espace intermédiaire entre la ville et le détroit, où déjà l'on touche les côtes espagnoles. Ce lieu est significatif de l'ici et de l'ailleurs. Surtout, il représente bien un vide mental, une rupture avec la tradition et l'habitude, qui en fait « l'observatoire des rêves ${ }^{23}$ ». De quels rêves? Bien entendu, du rêve de partir. De passer d'un lieu à l'autre, quitte à se perdre au sens propre dans la mort, le non-lieu absolu. La noyade est envisagée avec les yeux de ceux qui sont déjà perdus dans les « limbes du haschisch ${ }^{24} »$, et les limbes sont, précisément et d'un point de vue théologique, un lieu d'attente, entre la vie et la mort. Le territoire de la vie se déterritorialise en espace de la mort habité par la créature qui donne son nom à ce chapitre : "Toutia », araignée dévoreuse du corps des migrants clandestins noyés dans la mer. Dans la rêverie d'Azel, assis dans le Café Hafa, le centre urbain de Tanger

21. Ibid., p. 16-17.

22. Michel de Certeau, op. cit., p. 153.

23. Tahar Ben Jelloun, Partir, op. cit., p. 11. La citation complète est : « À Tanger, l'hiver, le café Hafa se transforme en un observatoire des rêves et de leurs conséquences. » 24. Ibid. 
se déplace, et il s'oriente mentalement dans un autre lieu, un autre centre, qui est « vers le milieu de la $\operatorname{mer}^{25}$ ».

Ce « cercle vert » complète et enserre la ville de Tanger, comme le plus sûr moyen de définir sa nature mêlée et son absence de clôture :

Il sait que là, dans ce cercle précis, existe une frontière mobile, une sorte de ligne de séparation entre deux eaux, celles calmes et plates de la Méditerranée et celles véhémentes et fortes de l'Atlantique ${ }^{26}$.

Ainsi, lorsque le personnage principal réussit finalement à quitter la ville pour l'Espagne, il écrit une lettre « à son pays » où il évoque l'espace géographique de Tanger qu'il souhaite apercevoir une dernière fois, au décollage de l'avion dans une saisie à la fois panoramique et de surplomb. Mais dans sa lettre, l'espace de la ville et celui du pays se superposent car la corruption qu'il cherche à fuir en quittant Tanger règne dans tout le pays. Ainsi, la partie ne se distingue plus du tout, le fragment devient totalité et Tanger devient le Maroc. Mais la dilatation ne s'arrête pas aux frontières du Maroc puisque le personnage retrouve à Malaga et Madrid des quartiers qui ressemblent tellement à sa ville natale « qu'il a l'impression d'être revenu à Tanger dans les méandres du petit $\operatorname{Socco}^{27} \gg$. Sous 1'effet de la drogue, il croit même avoir été enlevé et " remis dans un camion partant au Maroc » avant de rentrer chez lui pour écrire une nouvelle lettre « à son cher pays » et déplorer que, où qu'ils soient, les Marocains recréent « la joutya, le souk de leur ville pour se retrouver entre $\operatorname{eux}^{28} \gg$.

Mais la duplication vaut aussi pour les étrangers. C'est le cas pour Miguel, le protecteur espagnol d'Azel, qui reproduit à Madrid le mode de vie qu'il mène Tanger : il porte les mêmes burnous blancs, continue à utiliser son amant comme serviteur le jour et organise à Madrid des fêtes qui n'ont rien à envier à celles de Tanger, tant par leur faste (profusion de fleurs et de nourriture) que par leur aspect international, si ce n'est que des Brésiliens remplacent les vieilles princesses russes. De ce fait les espaces tangérois, marocain et espagnol finissent par être indifférenciés et enchevêtrés de telle manière qu'ils dessinent une nouvelle spatialité qui s'appréhende en regard de la mondialisation et induisent d'autres pratiques de la ville qui inscrivent de la mobilité et du parcours dans l'espace du texte.

\section{Tanger comme ville transhumante}

Le Tanger de Partir est en effet traversé de parcours divers, certains sont localisables car ils s'effectuent légalement sous forme d'aller-retour entre le Maroc et l'Espagne : l'enjeu pour les Marocains est d'obtenir de

25. Ibid., p. 14.

26. Ibid.

27. Ibid., p. 88.

28. Ibid., p. 89. 
leur protecteur un visa pour quitter leur pays. Mais la plupart des parcours ne sont pas localisables car ils se lisent le plus souvent sous forme de traces. Certaines sont écrites, mais presque effacées, comme c'est le cas lorsque Miguel découvre le journal de son père, écrit en 1951, sous Franco, où il raconte son départ vers le Maroc, en barque, de nuit avec une dizaine d'autres clandestins fuyant l'Espagne vers Tanger. Dans ce cas, ce sont les Espagnols qui fuient leur pays, sur des bateaux de fortune, au risque de se noyer, et c'est Tanger qui incarne le rêve de liberté. On pourrait presque parler d'une hétérotopie qui inverse les signes du réel, s'il ne s'agissait pas d'un épisode historique qui participe effectivement à la mémoire de la ville que Tahar Ben Jelloun évoque dans Partir. Cependant de nombreux parcours ne laissent pas de traces car ils se font clandestinement dans le sens Maroc-Espagne : Tanger est alors un lieu de départ pour l'Europe où prospèrent de nombreux passeurs. Mais les parcours se font aussi dans le sens Afrique-Maroc et Tanger devient alors un lieu où transitent des Africains dont il est dit que « certains parmi eux se noieraient bientôt dans la nuit noire ${ }^{29}$ "), sans laisser de traces. Mais les parcours peuvent aussi se faire, tout aussi clandestinement, dans le sens nord-sud comme c'est le cas pour les riches ressortissants des pays du Golfe qui viennent à Malaga et s'offrent parfois « pour une simple soirée la traversée du détroit et s'enferment dans les suites des grands hôtels de Tanger, faisant venir de l'alcool, de la nourriture, des musiciens et des filles ${ }^{30} »$.

L'espace représenté dans Partir est ainsi traversé de parcours qui remettent en question le concept de ville comme un ensemble lisible et structuré pour lui substituer celui de pratiques urbaines qui sont de l'ordre de la transhumance, de la migration. Mais ces pratiques nouvelles, qui dessinent une ville transhumante, n'entrent pas en contradiction avec la représentation du Tanger littéraire mythique puisque la ville reste toujours la ville de tous les trafics (les passeurs ont remplacé les trafiquants d'armes), de toutes les corruptions et de toutes les prostitutions. De ce fait et pour conclure, on peut constater que de « Tanger-la-trahison » à Partir, la représentation que Tahar Ben Jelloun élabore de la ville ne remet pas en question le Tanger légendaire qui continue d'y perdurer. Tout au plus ajoute-t-il une nouvelle strate temporelle en prenant en compte dans Partir le phénomène de l'immigration, absent des représentations précédentes. De ce fait, la représentation de Tahar Ben Jelloun participe de l'hétérotopie tangéroise et peut se lire comme un lieu autre, fortement investi par l'imaginaire, un lieu qui appartient à la catégorie proposée par Michel Foucault :

[...] des sortes de contre-emplacements, sortes d'utopies effectivement réalisées dans lesquelles les emplacements réels, tous les autres emplacements réels que l'on peut trouver à l'intérieur de la culture sont à la fois

29. Ibid., p. 188.

30. Ibid., p. 82. 
représentés, contestés et inversés, des sortes de lieux qui sont hors de tous les lieux, bien que pourtant ils soient effectivement localisables ${ }^{31}$.

Ce qui permet à Tanger de fonctionner comme une contestation de l'espace mondialisé et uniformisé dans lequel nous vivons désormais en se détournant d'un réel qu'il prétend pourtant prendre en compte.

Christiane Albert

Université de Pau et des Pays de l'Adour Centre de recherches en poétiques et histoire littéraire

Marc Kober

Université Paris 13, Sorbonne Paris Cité

Pléiade / CENEL

31. Michel Foucault, « Des espaces autres », op. cit., p. 47. 